\title{
Implicações do processo de Acreditação Hospitalar no cotidiano de profissionais de saúde*
}

\author{
IMPLICATIONS OF HOSPITAL ACCREDITATION ON THE \\ EVERYDAY LIVES OF HEALTHCARE PROFESSIONALS
}

\author{
IMPLICANCIAS DEL PROCESO DE CREDIBILIDAD HOSPITALARIA EN \\ EL COTIDIANO DE PROFESIONALES DE SALUD
}

Bruna Figueiredo Manzo', Maria José Menezes Brito², Allana dos Reis Corrêa ${ }^{3}$

\section{RESUMO}

A fim de alcançar os mais elevados padrões assistenciais, são adotadas iniciativas voltadas para o processo de Acreditação Hospitalar, as quais impõem mudanças comportamentais, mobilização dos profissionais em busca de metas e objetivos propostos, além da melhoria permanente e contínua do atendimento prestado. Trata-se de um estudo de caso de natureza qualitativa que teve como objetivo analisar as implicações do processo de Acreditação Hospitalar no cotidiano de profissionais de saúde de um hospital privado em Belo Horizonte. Os sujeitos da pesquisa foram 34 profissionais de saúde, alocados em diferentes setores do hospital. Os dados foram coletados por meio de entrevistas semi-estruturadas e analisados por meio da técnica de análise de conteúdo. Observou-se que o processo de Acreditação se configura como uma possibilidade de crescimento e de desenvolvimento da capacidade crítica dos atores envolvidos. Contudo, as exigências impostas por este processo desencadeiam stress profissional e sobrecarga de trabalho.

\section{DESCRITORES}

Hospitais

Acreditação

Pessoal de saúde

Qualidade da assistência à saúde

Equipe de enfermagem

\begin{abstract}
Hospital accreditation processes should be adopted to achieve the highest standards of healthcare. This process requires behavioral changes and mobilization of professionals in efforts to achieve goals and objectives, in addition to continuous improvement of healthcare. This qualitative case study was performed with the objective of analyzing the implications of hospital accreditation on the everyday lives of healthcare workers in a private hospital in Belo Horizonte. Subjects were 34 healthcare professionals from different sectors of the hospital. Data were collected through semi-structured interviews and subjected to content analysis. It was observed that the accreditation process was designed as an opportunity for developing and improving the critical thinking capacity of the subjects involved. However, the requirements imposed by this process may trigger occupational stress and cause work overload.
\end{abstract}

\author{
DESCRIPTORS \\ Hospitals \\ Accreditation \\ Health personnel \\ Quality of health care \\ Nursing, team
}

\begin{abstract}
RESUMEN
Apuntando a alcanzar los más elevados patrones de atención, se adoptan iniciativas orientadas al proceso de Credibilidad Hospitalaria, las cuales imponen cambios comportamentales, movilización de los profesionales tras las metas y objetivos propuestos, además de la mejora constante y continua en la atención brindada. Estudio de caso cualitativo, que objetivó analizar las implicancias del proceso de Credibilidad Hospitalaria en el cotidiano de profesionales de salud de un hospital privado en Belo Horizonte. Los sujetos de la investigación fueron 34 profesionales de salud, ubicados en diferentes sectores del hospital. Los datos se recogieron mediante entrevistas semiestructuradas y fueron analizados a través del análisis de contenido. Se observó que el proceso de Credibilidad se configura como una posibilidad de crecimiento y desarrollo de la capacidad crítica de los actores involucrados. Así y todo, las exigencias impuestas por este proceso, desencadenan estrés profesional y sobrecarga laboral.
\end{abstract}

\footnotetext{
* Extraído da dissertação "O processo de Acreditação Hospitalar na perspectiva de profissionais de saúde", Escola de Enfermagem da Universidade Federal de Minas Gerais, 2009. ${ }^{1}$ Enfermeira. Mestre em Enfermagem. Professora Assistente do Curso de Enfermagem da Pontifícia Universidade Católica de Minas Gerais. Membro do Núcleo de Pesquisa Administração em Enfermagem da Escola de Enfermagem da Universidade Federal de Minas Gerais. Belo Horizonte, MG, Brasil. brunaamâncio@yahoo.com.br ${ }^{2}$ Enfermeira. Doutora em Enfermagem. Professora Adjunta do Departamento de Enfermagem Aplicada da Escola de Enfermagem da Universidade Federal de Minas Gerais. Belo Horizonte, MG, Brasil. brito@enf.ufmg.br ${ }^{3}$ Enfermeira. Mestre em Enfermagem. Professora Assistente do Departamento de Enfermagem Básica da Escola de Enfermagem da Universidade Federal de Minas Gerais. Belo Horizonte, MG, Brasil. allanareiscorrea@gmail.com
} 


\section{INTRODUÇÃO}

A sociedade contemporânea se transforma de forma rápida e profunda. Buscando adequar-se às mudanças vividas pela sociedade contemporânea e, ao mesmo tempo, atender às demandas de uma clientela cada vez mais exigente, gestores dos serviços de saúde têm adotado novas posturas que impõem o incremento do nível de qualidade. Nessa perspectiva, temos o atual cenário brasileiro no contexto de uma economia globalizada onde as instituições não mais suportarão os custos relacionados com a má-qualidade, ou seja, gastos elevados com morbidade e mortalidade crescentes, despesas com retrabalho e dispêndios com processo ineficiente; ademais, chama a atenção para o comprometimento da imagem da organização perante a sociedade, além da perda de clientes e de mercado $^{(1)}$.

Considerando a necessidade de desenvolvimento de estratégia de incremento da qualidade dos serviços de saúde, o Programa de Acreditação Hospitalar é um procedimento de avaliação dos recursos institucionais, de forma periódica, voluntária, racionalizada, ordenadora e, principalmente, de educação continuada dos profissionais, com o intuito de garantir a qualidade da assistência por meio de padrões previamente aceitos ${ }^{(2)}$. A Acreditação deve ser entendida, portanto, em duas dimensões. A primeira, como um processo educacional, que leva as instituições prestadoras de serviços de assistência em saúde, especialmente os profissionais de saúde, a adquirirem a cultura da qualidade para implementação da gestão de excelência, fundamental para o processo. A segunda dimensão se refere ao processo de avaliação e certificação da qualidade dos serviços, analisando e atestando o grau de desempenho alcançado pela instituição de acordo com padrões pré-definidos ${ }^{(3)}$.

Esse movimento em direção à qualidade refletiu a necessidade de mudanças fundamentais nas organizações, uma vez que a valorização dos indivíduos e a atenção às relações sociais tornam-se imprescindíveis para a aquisição do título de excelência. Nesse cenário, os profissionais da saúde emergem como o cerne da política de qualidade, tendo papel essencial na garantia e na manutenção do processo. Por meio do compromisso com a política da qualidade estabelecida pela instituição, os profissionais se engajam e reforçam a cultura de melhoria centrada no cliente e na determinação de executar serviços que atendam os requisitos da Organização Nacional de Acreditação (ONA), de forma segura e com excelência. $O$ grande diferencial das organizações está no âmbito da qualidade de pessoal e no desempenho profissional de seus recursos humanos. A tecnologia e a estrutura das organizações po- dem ter um pequeno significado se as pessoas não se sentirem comprometidas com a qualidade, sobretudo no que se refere ao atendimento das necessidades dos clientes ${ }^{(4)}$.

$O$ estudo da qualidade envolve, além de um conjunto de técnicas, uma dimensão mais ampla de ações do processo social, cujos elementos principais para o seu desenvolvimento são aqueles que atuam e exercem seu papel nesse processo(5). Todo esforço de melhoria de uma organização deve começar por meio do enfoque dos profissionais no que tange à educação, desenvolvimento de habilidades, formação de consciência responsável, treinamentos para o trabalho em equipe e desenvolvimento da concepção ética do trabalho ${ }^{(4)}$.

Observa-se, portanto, que a implementação do sistema de qualidade não é tarefa simples, mas contrariamente, se apresenta como um grande desafio a ser enfrentado pelos gestores e profissionais que buscam adequar seus processos de trabalho a excelência no atendimento.

Espera-se, portanto, que este estudo possa contribuir para que as equipes de saúde reflitam sobre o processo da qualidade, a responsabilidade social, as mudanças de hábitos e dos conceitos embutidos no processo de Acreditação Hospitalar. Uma vez reconhecidas as implicações do sistema de qualidade pelos próprios profissionais, mais fácil será o caminho para a manutenção de todo o processo e a conquista da excelência no atendimento.

O objetivo deste estudo consiste em analisar as implicações do processo de Acreditação no cotidiano dos profissionais de saúde.

\section{REFERENCIAL TEÓRICO}

A preocupação com a qualidade, presente na saúde desde Hipócrates, torna-se mais intensa no século XX. Expressivas considerações foram tecidas por um pesquisador ${ }^{(6)}$, em 1998, a respeito dos diferentes modelos de qualidade que explicitam as peculiaridades da transposição da qualidade para a área de saúde. Nesse contexto o autor apontou a análise sistemática de seis atributos-chave. O primeiro diz respeito à eficácia como habilidade em atingir os melhores resultados na assistência por meio dos melhores cuidados. O segundo atributo trouxe a idéia de eficiência, que consiste na diminuição de custos da assistência sem diminuir as melhorias alcançadas. $O$ terceiro remete ao equilíbrio entre os custos e efeitos dos cuidados na assistência. O quarto incide na aceitabilidade do serviço conforme as expectativas dos pacientes e familiares. O quinto atributo propõe a conformidade com os conceitos sociais expressos em princípios éticos, valores, normas, regulamentos e leis. E por fim, esse autor refere-se à equidade na assistência à saúde como último 
atributo. Sendo assim, entre as estratégias para a garantia e melhoria da qualidade está a influência do desempenho profissional e do aprimoramento do modelo de gestão(6).

Desde o seu surgimento, os hospitais vêm passando por diversas mudanças, visando principalmente à melhoria da qualidade da assistência. Grandes avanços tecnológicos ocorreram e paralelamente a esses, novas formas de reorganização e reestruturação se fizeram necessárias nessas instituições ${ }^{(1)}$. Alguns autores ressaltam que o momento atual é permeado por imensas transformações dentre as quais se destacam a globalização da economia, o custo crescente dos serviços, o aumento de tecnologias e progresso da ciência, os avanços da informática, a produção e difusão de conhecimento técnico-científico ${ }^{(7)}$.

As instituições hospitalares tornaram-se mais complexas, havendo maior necessidade em buscar inovações de produtos e serviços para se manter em um mercado cada vez mais competitivo. Diante disso, as mudanças eram inevitáveis e necessárias à sobrevivência dessas instituições ${ }^{(8)}$.

A criação de instrumentos destinados à melhoria da qualidade da assistência na saúde tornou-se um fenômeno universal, deixando de ser um mero conceito teórico para ser uma realidade cuja essência é garantir a sobrevivência das empresas e dos setores de produção de bens e serviços. Ademais, a avaliação constitui um pilar fundamental de garantia da assistência na saúde e é entendida como sendo um instrumento da gestão de serviços de saúde necessário para mensurar os esforços da organização, qualidade dos serviços prestados, bem como sua utilidade e relevância social. Nesse contexto, a Acreditação Hospitalar se apresenta como um dos métodos de avaliação dos recursos das organizações de saúde, voluntário, periódico e reservado, que tende a garantir a qualidade da assistência por meio de padrões pré-estabelecido( ${ }^{(9)}$.

A Acreditação Hospitalar configura-se como uma metodologia desenvolvida para apreciar a qualidade da assistência oferecida em todos os serviços de um hospital. Tem como base a avaliação dos padrões de referência desejáveis, construídos por peritos da área e previamente divulgados, e nos indicadores ou instrumento que o avaliador emprega para constatar os padrões que estão sendo analisados. A solicitação da Acreditação pela instituição é um ato voluntário, periódico, espontâneo, reservado e sigiloso em que se pretende obter a condição de acreditada de acordo com padrões previamente aceitos, na qual é escolhida a instituição acreditadora que desenvolverá o processo de Acreditação(10).

Nos últimos anos, percebem-se importantes modificações na área da saúde no que se refere à necessidade de alcançar uma maior competitividade e conquistar no- vos mercados. O aumento crescente nos custos dos cuidados oferecidos, a necessidade de atender a expectativa e direitos do cliente, a preocupação com a garantia da segurança de atendimento são aspectos que exigem dos profissionais de saúde uma mudança na forma de gerir a saúde, sendo inovadores e, além de tudo, mostrando excelência no seu trabalho. Para garantir essa excelência e acompanhar os avanços, os hospitais sentiram a necessidade de criar métodos avaliativos para a prestação de seus serviços ${ }^{(11)}$.

O Programa Brasileiro de Acreditação Hospitalar visa o amplo conhecimento a respeito de um processo permanente de melhoria da qualidade assistencial, mediante a avaliação periódica do serviço. Para isso instituiu, no âmbito hospitalar, mecanismos para avaliação e aprimoramento contínuos da qualidade da assistência prestada. Sua concretização ocorreu mediante a elaboração do Manual Brasileiro de Acreditação Hospitalar (MBAH), o qual aborda os benefícios que as organizações de saúde obtêm ao se inserirem no processo de Acreditação Hospitalar, uma vez que a instituição demonstrará

responsabilidade e comprometimento com a segurança, com a ética profissional, com os procedimentos que realiza e com a garantia da qualidade do atendimento à população( ${ }^{(8,12)}$.

O Manual Brasileiro de Acreditação Hospitalar é um instrumento de avaliação da qualidade institucional, o qual é composto de seções e subseções. As seções representam os serviços, setores ou unidades com características semelhantes para que a instituição seja avaliada segundo uma consistência sistêmica; as subseções tratam do escopo de cada serviço, setor ou unidade, segundo três níveis, do mais simples ao mais complexo, sempre com um processo de incorporação dos requisitos anteriores de menor complexidade ${ }^{(8)}$.

A lógica das subseções é a de que todas possuem o mesmo grau de importância dentro do processo de avaliação. Cada subseção é composta por padrões que procuram avaliar estrutura, processo e resultado dentro de um único serviço, setor ou unidade sendo que cada padrão é constituído por uma definição e uma lista de itens de orientação que auxiliam na identificação do que se busca avaliar e na preparação das instituições prestadoras de serviço de saúde para o processo de Acreditação. Um nível superior só é alcançado quando o anterior tiver sido atingido ${ }^{(9)}$.

Os padrões exigidos e verificados na avaliação são divididos por níveis 1,2 e 3, com seus princípios norteadores e por padrões, sendo que cada padrão representa uma definição e uma lista de verificação que permite a identificação da sua necessidade e a concordância com o padrão estabelecido: 
Nível 1: Atende aos requisitos formais, técnicos e de estrutura para a sua atividade conforme legislação correspondente; identifica riscos específicos e os gerencia com foco na segurança. Princípio: Segurança.

Nível 2: Gerencia os processos e suas interações sistemicamente; estabelece sistemática de medição e avaliação dos processos; possui programa de educação e treinamento continuado, voltado para a melhoria de processos. Princípio: Organização (Processos).

Nível 3: Utiliza perspectivas de medição organizacional, alinhadas às estratégias e correlacionadas aos indicadores de desempenho dos processos; dispõe de sistemática de comparações com referenciais externos pertinentes, bem como evidências de tendência favorável para indicadores; apresenta inovações e melhorias implementadas, decorrentes do processo de análise-crítica. Princípio: Excelência na Gestão (Resultados) ${ }^{(8)}$.

\section{MÉTODO}

Trata-se de um estudo de caso de abordagem qualitativa. Os estudos qualitativos são aplicáveis ao estudo da história, das relações, das representações, das crenças, das percepções e das opiniões, produtos das interpretações que os sujeitos sociais fazem a respeito de como vivem, constroem seus artefatos, sentem e pensam ${ }^{(13)}$. Quanto ao estudo de caso, o mesmo apresenta-se como estratégia adequada quando se trata de questões nas quais estão presentes fenômenos contemporâneos inseridos em contextos da vida real e podem ser complementados por outras investigações de caráter exploratório e descritivo. O estudo de caso é utilizado como estratégia de pesquisa nos estudos organizacionais e gerenciais, contribuindo, de forma inigualável, para a compreensão de fenômenos complexos, nos níveis individuais, organizacionais, sociais e políticos e permitindo a preservação das características significativas dos eventos da vida real(14).

O cenário da pesquisa foi um hospital privado de médio porte, situado em Belo Horizonte e inaugurado em 2004 na perspectiva de ser referência de qualidade no atendimento para todos os hospitais da rede conveniada. Foram incluídos na pesquisa os setores de internação, Bloco Cirúrgico (BC) e Unidade de Cuidados Progressivos (UCP). O critério de inclusão dos setores mencionados foi o fato de existirem desde o momento da inauguração até a primeira auditoria externa, possibilitando aos trabalhadores uma experiência singular em relação ao processo de Acreditação.

A escolha dos sujeitos da pesquisa foi intencional e restrita aos profissionais que participaram do processo de Acreditação Hospitalar desde a primeira auditoria e que prestavam atendimento direto e contínuo aos usuários do serviço. Foram incluídos 34 profissionais de saúde sendo nove enfermeiros, 15 técnicos de enfermagem, seis médicos, um fonoaudiólogo e três fisioterapeutas, sendo a maioria $(88,2 \%)$ do sexo feminino. Quanto ao tempo de permanência na instituição, $64,7 \%$ dos entrevistados possuíam de 3 a 4 anos e 67,6\% eram profissionais alocados na UCP. Foram excluídos os profissionais com menos de três anos na instituição devido ao fato de não terem vivenciado o processo da Acreditação desde a primeira auditoria.

Preliminarmente à fase de coleta de dados o projeto foi autorizado pelo hospital e aprovado pelo comitê de ética em pesquisa da UFMG, com o Protocolo de aprovação ETIC n. 2567/08. Os sujeitos foram informados sobre o objetivo, justificativa e finalidades do estudo, atendendo a Resolução n. 196/96 do Conselho Nacional de Saú$\mathrm{de}^{(15)}$, que trata dos aspectos éticos da pesquisa envolvendo seres humanos. Todos os sujeitos que concordaram em participar do estudo assinaram o Termo de Consentimento Livre e Esclarecido. As entrevistas foram realizadas por meio de roteiro semi-estruturado entre os meses de setembro a novembro de 2008.

As entrevistas foram realizadas até que se alcançasse a saturação de informações, a qual pode ser definida como

a suspensão de inclusão de novos participantes quando

os dados obtidos passam a apresentar, na avaliação do pesquisador, certa redundância ou repetição, não sendo considerado relevante persistir na coleta de dados ${ }^{(16)}$.

Para manter o anonimato dos profissionais, as entrevistas foram identificadas pelas siglas de cada profissão e por um número referente de representante de cada categoria profissional, sendo E1 a E9 para os enfermeiros, T1 a T15 para os técnicos de enfermagem, M1 a M6 para médicos, F1 para a fonoaudiólogo e FT1 a FT3 para fisioterapeutas. Os dados foram submetidos à Técnica Análise de Conteúdo que busca alcançar uma interpretação mais profunda do fenômeno além de ultrapassar o alcance meramente descritivo do conteúdo manifesto da mensagem $^{(17)}$. Essa técnica se desdobra em três fases distintas: pré-análise, exploração do material e tratamento dos resultados o que permite a classificação e agregação dos achados de acordo com o problema explicitado ${ }^{(13)}$. Para tanto o texto foi recortado em unidades de registro sendo estabelecidas categorias temáticas, quais sejam: a face nebulosa da acreditação e a acreditação como possibilidade de crescimento e satisfação no trabalho.

\section{RESULTADOS}

\section{Implicações do processo de Acreditação para os profissionais de saúde}

Os depoimentos dos profissionais entrevistados nos permitiram evidenciar aspectos distintos do processo de Acreditação Hospitalar e de sua influência no cotidiano de trabalho desses profissionais. Observou-se, portanto, que a Acreditação Hospitalar é influenciada pelo nível de motivação e envolvimento dos trabalhadores e, ao mesmo tempo, influencia de forma significativa, o contexto de 
trabalho dos profissionais da saúde. Nessa perspectiva, foram identificados aspectos positivos e negativos deste processo, originando as seguintes categorias temáticas: $A$ face nebulosa da Acreditação e A Acreditação como possibilidade de crescimento e satisfação no trabalho.

\section{A face nebulosa da Acreditação}

Em relação aos funcionários do hospital pesquisado, foram observadas percepções distintas e, muitas vezes ambíguas em relação ao processo de acreditação em seu cotidiano de trabalho. Assim, por um lado foram destacados aspectos positivos, frequentemente relacionados com a possibilidade de crescimento pessoal e de valorização do currículo, alcançados mediante a realização de cursos de capacitação e atualização. Por outro lado, os aspectos negativos decorrem do estresse e da cobrança advindos das demandas impostas pelos processos de certificação, o que gera o sentimento de desmotivação, conforme ilustrado:

\begin{abstract}
De certa forma, o funcionário cresce, porque ele deixa de se envolver só com a prescrição médica e seguir prescrição, e ele começa a criar um tipo de pensamento também, crítico. Mas ao mesmo tempo, eu vejo que ele tem que se dividir, se desdobrar. Então, ele tem que ficar um tempo se dedicando à ONA, às folhas, à checagem e a toda burocratização de assistência aqui, e ela é dispensada. Ela é muito pesada, e nisso o paciente perde, e o funcionário sai daqui estressado, porque ele tem sempre a sensação eu não dou conta de fazer tudo. Então eu acho que isso desmotiva o funcionário em longo prazo (E3).
\end{abstract}

...o negativo da acreditação é o estresse mesmo (T5).

Em relação ao posicionamento dos entrevistados sobre os aspectos negativos, alguns autores afirmam que as mudanças nas organizações exigem dos trabalhadores alterações em suas estruturas de crenças e valores pessoais, levando-os a vivenciar situações de perda de referencial, medo e angústia como respostas psicológicas a situações ameaçadoras $^{(5)}$. Ainda nessa perspectiva, o processo de Acreditação foi referenciado como uma situação de constante cobrança e desencadeadora de estresse para os profissionais envolvidos, sem nada lhes favorecer, apesar de ser benéfico para o cliente, como evidencia o trecho do depoimento a seguir:

Profissionalmente eu acho que exige uma cobrança muito grande em relação a tudo para a gente. Visa à qualidade do cliente externo e esquece a qualidade do cliente interno (M4).

A falta de valorização também é uma queixa frequente dos sujeitos da pesquisa. Há um sentimento de pouco prestigio diante do grande desafio que ocorre no dia-a-dia, já que os erros são notados e criticados, ao passo que os elogios em face das metas e das vitórias conquistadas não apresentam grandes repercussões, conforme ilustrado:
Eu acho que profissional se sente estressado com tanta cobrança, a todo o momento ele sempre é cobrado por um erro, às vezes ele não tem tanto elogio de alguma coisa que ele fez bem feita. São sempre os erros que estão sendo buscados (E9).

Nota-se que os profissionais de saúde se posicionam em relação a um cenário permeado por cobrança e pressão, cabendo ressaltar que tal situação, apesar de inerente a qualquer sistema que utiliza de avaliação contínua, pode ser suavizada pelo incremento de estratégicas que busquem a valorização dos sujeitos envolvidos, a fim de proporcionar uma continuidade e maior adesão à gestão de qualidade. Também merece destaque o fato de alguns funcionários terem associado a proposta de aquisição do título com o aumento da remuneração. Tal associação encontra-se relacionada à necessidade de engajamento dos trabalhadores aos objetivos do hospital, ao aumento das exigências e do estresse, somados, à melhoria do atendimento decorrente da melhor capacitação, conforme observado no depoimento a seguir:

Para nós profissionais não faz diferença não, não mudou nada. Porque você ganhou o título não tem um aumento de salário. Não melhora. Com ou sem ONA não muda nada.

Acho que é pior ter ONA: a cobrança é muito grande (FT1).

Em um processo de melhoria da qualidade deve-se pensar em um sistema de recompensas e de reconhecimento dos esforços das pessoas que contribuem com o serviço de excelência. Os atos de esforço estimulam e motivam as pessoas quando devidamente recompensados, renovando o compromisso com a qualidade e com a excelência dos serviços prestados ${ }^{(4)}$.

\section{A Acreditação como possibilidade de crescimento $e$ satisfação no trabalho}

No que se refere à percepção positiva, observou-se que a acreditação desencadeia, nos profissionais, sentimentos de orgulho, satisfação e reconhecimento. Esses sentimentos encontram-se relacionados ao compartilhamento da responsabilidade pela conquista do título e pela valorização do hospital. O orgulho deriva da sensação de 'pertencimento' a uma empresa que se admira, de identificação de seus valores pessoais com os valores da organização ${ }^{(5)}$. Os depoimentos explicitam essa idéia:

Eu acho que a gente fica muito satisfeito quando passa a auditoria, você vê que o hospital foi acreditado e que a gente está envolvido, e que isso lá fora vai fazer a diferença para a gente (T15).

Você trabalha lá no hospital $X$ que é acreditado, nossa que bacana, ouvi falar que lá é muito bom, então você fica até orgulhoso (M1).

Algumas características, segundo os autores, são fontes de orgulho para os trabalhadores, quais sejam:

a variedade das tarefas que exige uma variedade de competências; a identidade do trabalho que permite realizar 
algo percebido como significativo, tangível e identificável; e o significado do trabalho que é sua capacidade de ter um impacto positivo sobre o bem-estar de outras pessoas ${ }^{(18)}$.

Ilustrando o ponto de vista dos autores, os profissionais entrevistados expressam a segurança proporcionada pelo processo de Acreditação, uma vez que o funcionário inserido em um serviço acreditado sente-se melhor preparado para atender as necessidades dos seus clientes, à medida que pode oferecer recursos materiais, técnicos e humanos de maior complexidade, além de manter o nível de qualidade pela padronização de rotinas e uma maior organização, o que pode ser evidenciado neste trecho:

Para os profissionais, eu acho que assim a partir do momento que se padronizam certas coisas, isso acaba sendo benéfico para o funcionário por que é um ambiente melhor para trabalhar e mais seguro, do que em novas instituições que não têm (E4).

O processo da Acreditação promove o incremento da melhoria das condições de trabalho pela segurança adquirida, bem como proporciona a estabilidade do clima organizacional entre os profissionais de saúde, ao mesmo tempo em que estabelece um ambiente de trabalho mais prazeroso e propício para fortalecimento das relações humanas. A melhoria nas condições de trabalho favorece a relação de crescimento, de entusiasmo e de prazer dos funcionários, gerando motivação para vivenciar futuras experiências e desenvolver o trabalho de maneira eficiente, com qualidade, em um ambiente de satisfação e alegria. Assim, o programa de Acreditação contribui para a melhoria do clima organizacional, estimulando o companheirismo entre colaboradores dos serviços, visto que trabalhar em um hospital acreditado e participar das atividades relacionadas à avaliação cria motivação no pessoal. A fala a seguir contempla essa idéia:

O hospital vai estar buscando que o clima seja legal para aquele profissional também, que entre a clientela interna, exista um clima, um relacionamento legal, preocupar também com a satisfação do trabalhador, com as condições de trabalho dele (T3).

Percebe-se que, ao atuar em um cenário de transformações no contexto hospitalar, profissionais de diferentes categorias (re) significam suas ações cotidianas, incluindo o fazer e o relacionar-se com. Estudos organizacionais têm evidenciado que a motivação e satisfação no trabalho ultrapassam a remuneração ou benefícios recebidos, efetivando-se por meio de um conjunto de fatores objetivos e subjetivos. Entre os fatores subjetivos destacamos o sentido atribuído ao trabalho para quem o executa. Para ter sentido, o trabalho deve contemplar, na sua realização, a prática e desenvolvimento de competências, o exercício de julgamentos e livre arbítrio, a informação sobre seu desempenho e a oportunidade de se ajustar ${ }^{(4)}$. No âmbito da Acreditação, a qualidade é tudo que agrega valor ao trabalho ou às relações e está diretamente ligada à produtividade da instituição, envolvendo o relacionamento humano e o desenvolvimento profissional e pessoal(5).

\section{DISCUSSÃO}

Os resultados descritos no presente estudo indicam aspectos positivos e negativos do processo de Acreditação Hospitalar que influenciam diretamente no cotidiano de trabalho desses profissionais. Os profissionais não visualizam de maneira uniforme a influência do processo da Acreditação, alguns funcionários conseguem perceber o processo apenas de forma positiva, outros o abordam somente com visão negativa. Porém, há aqueles que conseguem ponderar sobre a Acreditação, dela extraindo os aspectos negativos e os pontos de sucesso do processo.

Para os profissionais que veem o processo positivamente, a Acreditação traz orgulho e satisfação, tendo em vista a sensação de responsabilidade pela conquista do título e pela valorização do hospital. Outros aspectos positivos mencionados estão, muitas vezes, relacionados com a possibilidade de crescimento pessoal e a valorização do currículo com o aprendizado. Revela-se, também a questão da segurança proporcionada pelo processo de Acreditação ao profissional de saúde. Os sujeitos da pesquisa abordaram que o funcionário inserido em um serviço acreditado sente-se melhor preparado para atender as solicitações dos seus clientes, além de manter o nível de qualidade pela padronização de rotinas e uma maior organização.

Dentre os pontos negativos, por sua vez, nota-se que os profissionais de saúde se veem em um cenário permeado por cobrança, pressão, estresse, falta de valorização e reconhecimento. Vale ressaltar que tal situação, apesar de inerente a qualquer sistema que utiliza de avaliação contínua e busca melhoria contínua, pode ser suavizada pelo incremento de estratégicas que busquem a valorização dos sujeitos envolvidos, a fim de proporcionar uma continuidade e maior adesão à gestão de qualidade.

No confronto entre as razões positivas e os argumentos negativos apresentados pelos entrevistados no âmbito das implicações do processo de Acreditação para o meio profissional, observa-se que a valorização e o conhecimento adquiridos por cada indivíduo superam o stress e a cobrança, já que é impossível conquistar melhorias sem o aumento da responsabilidade. Aliás, talvez os pontos negativos apresentados advenham da própria novidade, pois, o novo projeta insegurança e resistência imediatas, mas momentâneas. Assim é inegável que a recompensa voltada à valorização e ao reconhecimento do profissional, mediante elogios, palavras de apoio e outras formas de incentivo principalmente do ponto de vista financeiro, minimizaria a visão negativa apresentada pelo entrevistado.

Ressalta-se a constante necessidade de investimentos em educação permanente para a viabilização das mudanças nos processos internos e externos da organização e responder às demandas advindas dos protocolos de certificação e simultaneamente dos profissionais de saúde. Desta forma, a Acreditação pode representar uma importante estratégia de capacitação de pessoal no âmbito do hospital, o que reflete 
a atual tendência da gestão de pessoas na qual, deve-se promover a conciliação das expectativas organizacionais e dos trabalhadores.

\section{CONCLUSÃO}

A busca incessante pela melhoria da qualidade dos serviços prestados é o caminho para as instituições que lutam pela permanência no mercado competitivo e que prezam pelo atendimento de excelência. Por isso, os hospitais procuram novos modelos assistenciais e outras formas de gestão, a fim de alcançarem resultados capazes de otimizar recursos, incrementar o cuidado humanizado, além de garantir a melhoria do serviço oferecido. Nesse contexto, o programa de Acreditação Hospitalar surge como uma possibilidade de promover mudanças no cenário atual, desgastado pelo modelo tecnicista de atendimento. 0 novo processo de qualidade traz mudanças de hábitos, de valores e de comportamentos, impõe aos sujeitos envolvidos a ruptura do cuidado

\section{REFERÊNCIAS}

1. Maximiano ACA. Teoria geral da administração: da escola científica à competitividade em economia globalizada. $2^{\underline{a}}$ ed. São Paulo: Atlas; 2000. O enfoque da qualidade na administração; p. 248-62.

2. Schiesari LMC. Resultados de iniciativas de qualidade em hospitais brasileiros [tese doutorado]. São Paulo: Faculdade de Saúde Pública, Universidade de São Paulo; 2003.

3. Quinto Neto A, Bittar OJN. Hospitais: administração da qualidade e acreditação de organizações complexas. Porto Alegre: Dacasa; 2004.

4. Mezomo JC. Gestão da qualidade na saúde: princípios básicos. São Paulo: Loyola; 2001.

5. Bonato VL. Gestão em saúde: programas de qualidade em hospitais. São Paulo: Icone; 2007.

6. Donabedian A. The quality of health: how can it be assured? JAMA. 1998;260(12):1743-8.

7. D'Innocenzo M, Adami NP, Cunha ICKO. O movimento pela qualidade nos serviços de saúde e enfermagem. Rev Bras Enferm. 2006;59(1):84-8.

8. Brasil. Ministério da Saúde; Secretaria de Assistência à Saúde. Manual Brasileiro de Acreditação Hospitalar. 7ạ ed. Brasília; 2006.

9. Labbadia LL, Matsushita MS, Piveta VM, Viana TA, Cruz FSL. O processo de acreditação hospitalar e a participação da enfermeira. Rev Enferm UERJ. 2004;12(1):83-7.

10. Organização Nacional de Acreditação (ONA). Manual para Avaliação e Certificação de Organizações Prestadoras de Serviços Hospitalares. Brasília; 2004. mecanizado, bem como fomenta um ambiente organizacional de excelência.

Este estudo buscou aprofundar a reflexão sobre as implicações do processo de Acreditação para os profissionais de saúde, reforçando que para a implementação, a adesão e a manutenção do processo de qualidade nos hospitais é imprescindível, antes de tudo, conhecer a percepção daqueles sujeitos acerca da Acreditação.

Espera-se, portanto, que a partir do maior conhecimento sobre as implicações do processo de acreditação no cotidiano dos profissionais de saúde, barreiras sejam rompidas, comportamentos se alterem e o agir se qualifique. Almeja-se, além disso, que este estudo possa suscitar novas pesquisas sobre o tema, além de provocar nos profissionais de saúde, motivação e compromisso para promoverem as mudanças necessárias, bem como propiciar ao paciente uma melhor prestação de serviços, com um cuidado humanizado e de excelência.

11. Feldman LB, Gatto MAF, Cunha ICKO. História da evolução da qualidade hospitalar: dos padrões a Acreditação. Acta Paul Enferm. 2005;18(2):213-9.

12. Vituri DW, Matsuda LM. Content validation of quality indicators for nursing care evaluation. Rev Esc Enferm USP [Internet]. 2009 [cited 2010 July 15];43(2):429-37. Available from: http://www.scielo.br/pdf/reeusp/v43n2/en_a24v43n2.pdf

13. Minayo MCS. O desafio do conhecimento: pesquisa qualitativa em saúde. 10a ed. São Paulo: Hucitec; 2007.

14. Yin RK. Estudo de caso: planejamento e métodos. 3a ed. Porto Alegre: Bookman; 2005.

15. Conselho Nacional de Saúde. Resolução n. 196, de 10 de outubro de 1996. Dispõe sobre diretrizes e normas regulamentadoras de pesquisas envolvendo seres humanos. Bioética. 1996;4(2 Supl):15-25.

16. Fontanella BJB, Ricas J, Turato ER. Amostragem por saturação em pesquisas qualitativas em saúde: contribuições teóricas. Cad Saúde Pública. 2008;24(1):17-27.

17. Bardin L. Análise de conteúdo. Lisboa: Edições 70; 1977.

18. Tanure B, Carvalho Neto A, Andrade J. Fontes de tensão no olimpo empresarial brasileiro: tempo de menos, mudanças e sobrecargas demais, muito orgulho e o peso do teatro corporativo. In: Helal DH, Garcia FC, Honório LC, coordenadores. Gestão de pessoas e competência: teoria e pesquisa. Curitiba: Juruá; 2008. p. 16-39. 